\title{
The effect of a low dose of caffeine on exercise performance and thermoregulation during endurance exercise in the heat
}

\author{
R. E. Beaumont, D. Brown, B. Stephenson, C. Freeman and P. Watson \\ School of Sport, Exercise, and Health Sciences, Loughborough University, Leicestershire, UK
}

Despite a considerable amount of evidence supporting the use of caffeine prior to exercise in temperate conditions ${ }^{(1)}$, there is evidence to suggest that moderate to large doses of caffeine (i.e. $6-9 \mathrm{mg} / \mathrm{kg}$ ) ingested prior to exercise in the heat does not influence endurance performance ${ }^{(2)}$. This response was attributed to perturbations in thermoregulation, potentially limiting the positive central effects. It is unclear at present whether the provision of a low dose of caffeine affects performance and/or thermoregulation in the same way. Therefore, the aim of this study was to examine whether ingesting low doses of caffeine prior to exercise in a warm environment could improve endurance performance compared to placebo.

With approval from the local ethics committee, eight recreationally active males (mean data: age, $20 \mathrm{y}$; body mass, $80 \cdot 86 \mathrm{~kg}$; height, $1.79 \mathrm{~m} ; \mathrm{VO}_{2}$ Peak, $3.9 \mathrm{~L}$ ) undertook an incremental exercise test to determine maximal oxygen uptake, a familiarisation trial and three experimental trials (placebo [PLA], single dose caffeine [ $3 \mathrm{mg} / \mathrm{kg}$ body mass; CP], split dose caffeine [2 $\times 1.5 \mathrm{mg} / \mathrm{kg}$ body mass; CC]). Trials were undertaken in a double blind, randomised order. Volunteers completed ninety minutes of exercise $(60 \mathrm{~min}$ pre-load at $60 \%$ $\mathrm{VO}_{2}$ Peak followed by a 30 minute time trial [TT]) in environmental conditions of $30^{\circ} \mathrm{C}$ and $50 \% \mathrm{RH}$.

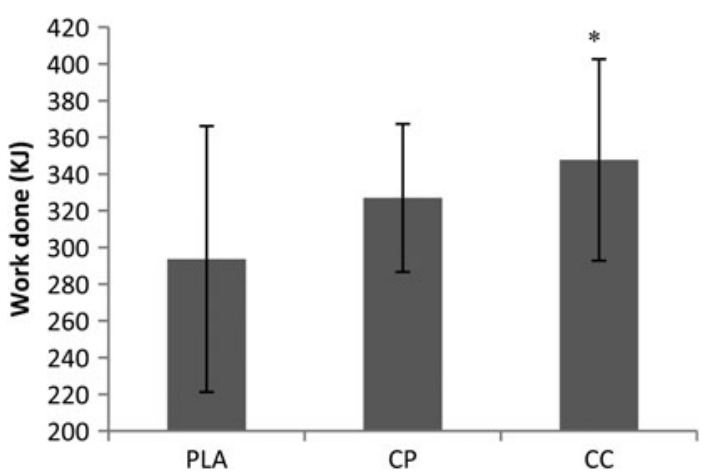

Fig. 1. Total work completed during time trial

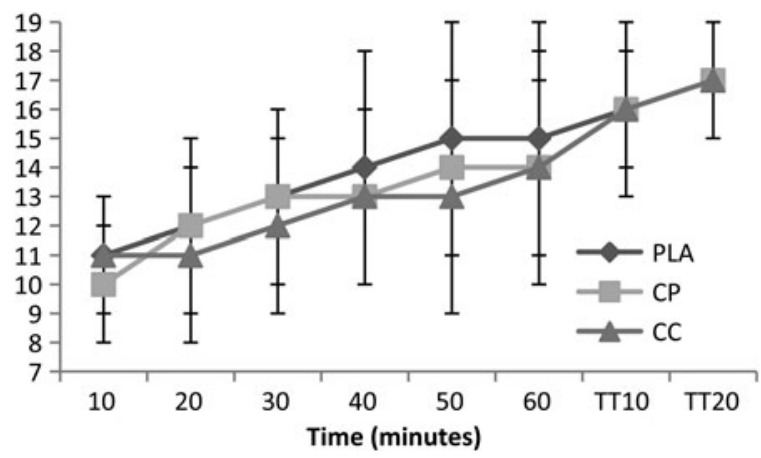

Fig. 2. Rating of perceived exertion. $* P=0.022$ compared to PLA. Main effect for trial (CC vs. PLA) $P=0.32$

Total work produced during the TT was greater in trial CC compared to PLA (Fig 1, $P=0.022)$. There was no statistical difference between CP and PLA $(P=0.318)$. Core temperature was not influenced by caffeine treatment $(P=0 \cdot 151)$, with mean values of 38.77 , 39.06 , and $38.80^{\circ} \mathrm{C}$ recorded at the end of the time trial in the PLA, CP, and CC trials, respectively $(P>0.05)$. Skin temperature was not influenced by caffeine treatment $(P=0.41)$. Despite an increase in performance, the $C C$ trial showed a reduced perception of effort than PLA (Fig 2, $P=0.34)$, with no differences in thermal stress apparent between trials $(P=0.517)$.

These data confirm the results of a recent study ${ }^{(3)}$ and lend support to the view that the provision of a low dose of caffeine may prove a prudent strategy to enhance endurance performance in the heat. It appears that lower doses do not appreciably alter core or skin temperature during exercise, perturbations which have previously been observed when higher doses have been consumed prior to exercise in the heat.

1. Doherty M and Smith PM (2004) Scan J Med Sci Sports 15, 69-78

2. Roelands B, Buyse L, Pauwels F et al. (2011) Eur J Appl Physiol 111, 3089-3095.

3. Pitchford NW, Fell JW, Leveritt MD et al. (2013) J Sci Med Sport 13, S1440-2440. 